\title{
Conservative discretization of multiphase flow with high density ratios
}

\author{
L. Jofre ${ }^{1}$, O. Lehmkuhl ${ }^{1,2}$, N. Balcázar ${ }^{1}$, J. Castro $^{1}$, J. Rigola ${ }^{1}$ \\ \& A. Oliva ${ }^{1}$ \\ ${ }^{1}$ Heat and Mass Transfer Technological Center, \\ Technical University of Catalonia, Spain \\ ${ }^{2}$ Termo Fluids S.L., Spain
}

\begin{abstract}
The computation of multiphase flows presenting high density ratios, where the fluids involved are considered immiscible, are of great importance for fundamental physics and industrial applications; such as the study of liquid-gas interfaces, wave motion, simulation of bubbly flows and atomization, injection in diesel engines, chemical processes and others.

This work presents and analyzes a collocated and staggered finite-volume mesh discretizations suitable for three-dimensional unstructured meshes, which are able to simulate immiscible multiphase flows with high density ratios. More over, these mesh schemes numerically conserve mass and momentum while minimize errors in the conservation of kinetic energy.

Keywords: collocated, conservation, multiphase, Navier-Stokes, staggered.
\end{abstract}

\section{Introduction}

Multiphase immiscible-phase flows with sharp density jumps are found in many industrial applications. For example, the study of fluid-fuel interactions, formation of bubbles and droplets, wave motion and others. This type of flows are usually referred as interfacial flows, since the contact of immiscible fluids or phases in motion produces a thin region that separates them called interface. Interfacial flows are governed by the unsteady Navier-Stokes equations in the variabledensity incompressibility limit. Over the years, the collocated and staggered mesh discretizations have stand out for the computation of the discrete Navier-Stokes equations. 
On the one hand, collocated mesh discretizations calculate velocity and pressure at cell centers while require particular interpolations and special mass fluxes at faces. On the other hand, staggered mesh schemes directly solve mass fluxes at faces and store pressure at cell centers. Then, cell-centered velocities need to be interpolated from face mass fluxes.

In the last decade, an important effort has been made to improve the stability and robustness of immiscible multiphase models by studying in detail the pressure Poisson matrix [1] and proposing complex face density interpolations [2]. Otherwise, numerical techniques used for the simulation of turbulent flow have evolved to discretely preserve kinetic energy [3-5] by using skew-symmetric formulations, at expenses of increasing the local truncation error. Hence, this work aims to introduce this conservation idea for the simulation of interfacial flow discretizating the momentum equations by means of symmetry-preserving collocated and staggered mesh schemes.

\section{Discrete Navier-Stokes equations}

Multiphase immiscible-phase flows are governed by the unsteady Navier-Stokes equations in the variable-density incompressibility limit, written in divergence form as

$$
\begin{gathered}
\nabla \cdot \mathbf{u}=0 \\
\frac{\partial(\rho \mathbf{u})}{\partial t}+\nabla \cdot(\rho \mathbf{u u})=-\nabla p+\nabla \cdot\left(\mu\left(\nabla \mathbf{u}+\nabla^{T} \mathbf{u}\right)\right)+\mathbf{S}
\end{gathered}
$$

where $\mathbf{u}, p$ and $\mathbf{S}$ represent velocity, pressure and a general source term. Density, $\rho$, and dynamic viscosity, $\mu$, are evaluated as the phase-volume fraction interpolation of the properties for each phase $k$, given as

$$
\rho=\sum_{k} C_{k} \rho_{k} \quad \text { and } \quad \mu=\sum_{k} C_{k} \mu_{k} .
$$

\subsection{Collocated mesh scheme}

The collocated mesh scheme calculates velocity and pressure fields at cell centers while needs particular interpolations and special mass fluxes at faces, in order to minimize the kinetic energy error and conserve mass exactly.

The velocity-pressure coupling of the momentum equation, Eq. 2, is solved by means of a classical fractional step projection method along with explicit timeadvancement, written as

$$
\begin{gathered}
\rho^{n+1} \mathbf{u}^{n+1}-\rho^{n+1} \mathbf{u}^{p}=-\Delta t \nabla p^{n+1}, \\
\rho^{n+1} \mathbf{u}^{p}=\rho^{n} \mathbf{u}^{n}-\Delta t\left[\nabla \cdot\left(\rho^{n} \mathbf{u}^{n} \mathbf{u}^{n}\right)-\nabla \cdot\left(\mu^{n}\left(\nabla \mathbf{u}^{n}+\nabla^{T} \mathbf{u}^{n}\right)\right)-\mathbf{S}^{n}\right],
\end{gathered}
$$

where superscript $n$ refers to time instant, $\mathbf{u}^{p}$ is the predictor velocity and $\Delta t$ is the time step. 
First, the predictor velocity is discretizated by integrating Eq. 5 over cell $c$ and applying the divergence theorem to its faces, $f \in F(c)$, giving

$$
\begin{aligned}
\rho_{c}^{n+1} \mathbf{u}_{c}^{p}= & \rho_{c}^{n} \mathbf{u}_{c}^{n}-\frac{\Delta t}{V_{c}} \sum_{f \in F(c)} \phi_{f}^{n} \hat{M}_{f}^{n} \\
& +\frac{\Delta t}{V_{c}}\left[\sum_{f \in F(c)} \mu_{f}^{n}\left[\left(\mathbf{u}_{n b}^{n}-\mathbf{u}_{c}^{n}\right) \frac{A_{f}}{\delta d_{f}}+\nabla^{T} \mathbf{u}_{f}^{n} \cdot \hat{\mathbf{n}}_{f} A_{f}\right]\right]+\Delta t \mathbf{S}_{c}^{n},
\end{aligned}
$$

where $V_{c}$ is the volume of cell $c, \phi_{f}$ is the convected face velocity, $\hat{M}_{f}$ is the face mass flux, $\hat{\mathbf{n}}_{f}$ is the outward-unit face normal, $A_{f}$ is the face surface, subscripts $c$ and $n b$ refer to the cell itself and the face-neighbor one, and length $\delta d_{f}$ is the normal-projected distance between centroids of cells.

Next, dividing Eq. 4 by density, $\rho^{n+1}$, multiplying by the divergence operator, applying the incompressibility condition, Eq. 1, and discretizating over cell $c$, yields a discrete Poisson equation

$$
\sum_{f \in F(c)} \frac{\hat{M}_{f}^{p}}{\rho_{f}^{n+1}}=\Delta t \sum_{f \in F(c)} \frac{1}{\rho_{f}^{n+1}}\left(p_{n b}^{n+1}-p_{c}^{n+1}\right) \frac{A_{f}}{\delta d_{f}}
$$

which solves the pressure field. When the solution of $p^{n+1}$ is obtained, $\mathbf{u}^{n+1}$ results from discretizating Eq. 4 over cell $c$ as

$$
\mathbf{u}_{c}^{n+1}=\mathbf{u}_{c}^{p}-\frac{\Delta t}{\rho_{c}^{n+1} V_{c}} \sum_{f \in F(c)} p_{f}^{n+1} \hat{\mathbf{n}}_{f} A_{f},
$$

where $p_{f}$ is the pressure interpolated to face $f$.

Notice that no specific interpolations for $\phi_{f}^{n}, \hat{M}_{f}^{p}, \rho_{f}^{n+1}$, and $p_{f}^{n+1}$ have been explained yet. Therefore, in order to fulfill the skew-symmetric requirement of the discrete convective operator, the convected face velocity is evaluated as $\phi_{f}^{n}=$ $\frac{1}{2}\left(\mathbf{u}_{c}^{n}+\mathbf{u}_{n b}^{n}\right)$ [4]. On the other hand, face predictor mass flux, density and pressure are calculated as $\hat{M}_{f}^{p}=\frac{1}{2}\left(\rho_{c}^{n+1} \mathbf{u}_{c}^{p}+\rho_{n b}^{n+1} \mathbf{u}_{n b}^{p}\right) \cdot \hat{\mathbf{n}}_{f} A_{f}, \rho_{f}^{n+1}=\frac{1}{2}\left(\rho_{c}^{n+1}+\rho_{n b}^{n+1}\right)$ and $p_{f}^{n+1}=\frac{1}{2}\left(p_{c}^{n+1}+p_{n b}^{n+1}\right)$, minimizing the kinetic energy conservation error as it will be demonstrated in Sec. 3 .

Finally, the mass-conserving evaluation of the face mass flux, $\hat{M}_{f}^{n+1}$, is defined as

$$
\hat{M}_{f}^{n+1}=\hat{M}_{f}^{p}-\Delta t\left(p_{n b}^{n+1}-p_{c}^{n+1}\right) \frac{A_{f}}{\delta d_{f}} .
$$


Then, if the predictor mass flux is evaluated as the semi-sum $\hat{M}_{f}^{p}=\frac{1}{2}\left(\rho_{c}^{n+1} \mathbf{u}_{c}^{p}+\right.$ $\left.\rho_{n b}^{n+1} \mathbf{u}_{n b}^{p}\right) \cdot \hat{\mathbf{n}}_{f} A_{f}$ and $\mathbf{u}^{p}$ is substituted using Eq. 8, Eq. 9 is rewritten as

$$
\begin{aligned}
\hat{M}_{f}^{n+1}= & \frac{1}{2}\left(\rho_{c}^{n+1} \mathbf{u}_{c}^{n+1}+\rho_{n b}^{n+1} \mathbf{u}_{n b}^{n+1}\right) \cdot \hat{\mathbf{n}}_{f} A_{f}-\Delta t\left(p_{n b}^{n+1}-p_{c}^{n+1}\right) \frac{A_{f}}{\delta d_{f}} \\
& +\Delta t\left[\frac{1}{2}\left[\frac{1}{V_{c}} \sum_{f \in F(c)} p_{f}^{n+1} \hat{\mathbf{n}}_{f} A_{f}+\frac{1}{V_{n b}} \sum_{f \in F(n b)} p_{f}^{n+1} \hat{\mathbf{n}}_{f} A_{f}\right]\right] \cdot \hat{\mathbf{n}}_{f} A_{f} .
\end{aligned}
$$

\subsection{Staggered mesh scheme}

The staggered mesh scheme stores pressure and other scalar quantities at cell centers while mass fluxes are distributed to cell faces. Each face stores only the mass flux, therefore, the cell-centered velocity vector has to be recovered from face normal components. This work extends the staggered mesh discretization developed by Perot [3], suitable for unstructured meshes, to variable-density flows.

Thus, integrating Eq. 4 and 5 over face $f$ control volume and taking a dot product with the face normal vector, $\mathbf{n}_{f}$, results in the discrete staggered form of the fractional step projection method

$$
\begin{gathered}
M_{f}^{n+1}=M_{f}^{p}-\Delta t\left(p_{b}^{n+1}-p_{a}^{n+1}\right) \frac{A_{f}}{\left(W_{a}+W_{b}\right)}, \\
M_{f}^{p}=M_{f}^{n}-\Delta t\left[W_{a}\left(\mathbf{c}_{a}-\mathbf{d}_{a}-\mathbf{s}_{a}\right)+W_{b}\left(\mathbf{c}_{b}-\mathbf{d}_{b}-\mathbf{s}_{b}\right)\right] \frac{A_{f} \cdot \mathbf{n}_{f}}{\left(W_{a}+W_{b}\right)} .
\end{gathered}
$$

Subscripts $a$ and $b$ refer to the two cells adjacent to face $f$ and $\mathbf{c}, \mathbf{d}$ and $\mathbf{s}$ are the non-volumetric cell-centered discretizations of convective, diffusive and source terms evaluated for each cell $c$ as

$$
\begin{gathered}
\mathbf{c}_{c}=\frac{1}{V_{c}} \sum_{f \in F(c)} \phi_{f}^{n} \hat{M}_{f}^{n}, \quad \mathbf{s}_{c}=\frac{1}{V_{c}} \mathbf{S}_{c}^{n} V_{c} \\
\mathbf{d}_{c}=\frac{1}{V_{c}} \sum_{f \in F(c)} \mu_{f}^{n}\left[\left(\mathbf{u}_{n b}^{n}-\mathbf{u}_{c}^{n}\right) \frac{A_{f}}{\delta d_{f}}+\nabla^{T} \mathbf{u}_{f}^{n} \cdot \hat{\mathbf{n}}_{f} A_{f}\right],
\end{gathered}
$$

where the convected face velocity, $\phi_{f}$, is evaluated as previously defined for the collocated formulation and length $\delta d_{f}$ is once again the distance between cells' nodes.

Next, dividing Eq. 4 by density, multiplying by the divergence operator, using the incompressibility condition and discretizing over cell $c$, gives the discrete Poisson equation already presented, Eq. 7. In this case no interpolation for the predictor mass flux is needed since it is given by Eq. 12 and $\delta d_{f}$ is now the distance between cells' circumcenters. When the solution of $p^{n+1}$ is calculated, Eq. 11 is used to obtain the face mass fluxes at instant $n+1, M_{f}^{n+1}$.

Finally, the staggered mesh scheme discretizes face mass fluxes in time, then, cell-centered velocities need to be interpolated from face normal components. 
Then, if a first-order approximation of the momentum field (constant $\rho \mathbf{u}$ ) is assumed, cell-centered velocities are defined as

$$
\mathbf{u}_{c}=\frac{1}{\rho_{c} V_{c}} \sum_{f \in F(c)} \mathbf{r}_{f}^{c} \hat{M}_{f}
$$

and $\mathbf{r}_{f}^{c}$ is the vector from cell circumcenter, $\mathbf{x}_{c}^{C C}$, to face centroid, $\mathbf{x}_{f}^{C G}$.

\section{Conservation properties}

\subsection{Mass conservation}

In the collocated case a special definition for the face mass flux, Eq. 10, has been developed in order to exactly conserve mass for each cell $c$. On the other hand, for the staggered case no interpolation of the face mass flux is needed since it is calculated at cell faces by definition of the scheme. Hence, in both cases the mass is locally conserved, consequently, global mass conservation, Eq. 15, equals zero since it is a summation of locally mass-conserving quantities, expressed as

$$
\int_{\Omega} \nabla \cdot \mathbf{u} d V=\sum_{c \in \Omega} \sum_{f \in F(c)} \hat{U}_{f} A_{f}=\sum_{c \in \Omega} \sum_{f \in F(c)} \frac{\hat{M}_{f}}{\rho_{f}}=0 .
$$

\subsection{Momentum conservation}

\subsubsection{Collocated momentum conservation}

Conservation of momentum is obtained by integrating Eq. 2 over the entire domain, which is transformed to a summation of integrals for each control volume that form the domain, converted to surface integrals by applying the divergence theorem and simplified by canceling interior fluxes, giving

$$
\begin{aligned}
& \sum_{c \in \Omega} \frac{d\left(\rho_{c} \mathbf{u}_{c}\right)}{d t} V_{c}+\sum_{f \in F(\partial \Omega)} \phi_{f} \hat{M}_{f}=-\sum_{f \in F(\partial \Omega)} p_{f} \hat{\mathbf{n}}_{f} A_{f} \\
& +\sum_{f \in F(\partial \Omega)} \mu_{f}\left[\left(\mathbf{u}_{f}-\mathbf{u}_{a}\right) \frac{A_{f}}{\delta d_{f}}+\nabla^{T} \mathbf{u}_{f} \cdot \hat{\mathbf{n}}_{f} A_{f}\right]+\sum_{c \in \Omega} \mathbf{S}_{c} V_{c},
\end{aligned}
$$

which states that the change in momentum is due to the fluxes through the boundary of the domain and the source terms.

\subsubsection{Staggered momentum conservation}

The primary quantity in staggered mesh schemes is the face mass flux. Thus, integrating Eq. 2 over face $f$ control volume and taking a dot product with the face normal vector, $\mathbf{n}_{f}$, gives the discretizated momentum equation for the face 
mass flux, $M_{f}$, written as

$$
\begin{aligned}
\left(W_{a}+W_{b}\right) \frac{d M_{f}}{d t} & +\left(W_{a} \mathbf{c}_{a}+W_{b} \mathbf{c}_{b}\right) A_{f} \cdot \mathbf{n}_{f}=-\left(p_{b}-p_{a}\right) A_{f} \\
& +\left(W_{a} \mathbf{d}_{a}+W_{b} \mathbf{d}_{b}\right) A_{f} \cdot \mathbf{n}_{f}+\left(W_{a} \mathbf{s}_{a}+W_{b} \mathbf{s}_{b}\right) A_{f} \cdot \mathbf{n}_{f} .
\end{aligned}
$$

Discrete staggered conservation of momentum is shown by multiplying Eq. 17 by the face normal vector, $\mathbf{n}_{f}$, and summing over all faces of the domain, $f \in$ $F(\Omega)$, which can be recasted to Eq. 16 by applying mathematical properties. Hence, the momentum conservation for the staggered scheme states that the change in momentum is due to the fluxes through the boundary of the domain and the source terms, as in the collocated scheme case.

\subsection{Kinetic energy conservation}

The transport equation for kinetic energy is derived from the momentum equation, Eq. 2, by taking the velocity dot product and assuming incompressible fluid. In this way, the kinetic energy can be shown to obey

$$
\frac{\partial\left(\frac{1}{2} \rho \mathbf{u} \cdot \mathbf{u}\right)}{\partial t}+\nabla \cdot\left[\mathbf{u}\left(\frac{1}{2} \rho \mathbf{u} \cdot \mathbf{u}\right)\right]=-\nabla \cdot(p \mathbf{u})+\nabla \cdot(\mu \mathbf{u} \times \omega)-\mu \omega \cdot \omega+\mathbf{S} \cdot \mathbf{u}
$$

where $\omega=\nabla \times \mathbf{u}$ is the vorticity. The important characteristic of this equation is that it is conservative except for the negative definite sink term, $\mu \omega \cdot \omega$, and the source term, $\mathbf{S} \cdot \mathbf{u}$. In the absence of external forces and viscosity, the kinetic energy is simply redistributed but not created or destroyed.

\subsubsection{Collocated kinetic energy conservation}

In order to investigate the collocated conservation of kinetic energy, the momentum equation, Eq. 2, is discretizated over the whole domain and multiplied by the velocity vector, $\mathbf{u}$. Then, the resulting equation can be transformed to a summation of surface integrals for each cell $c$, written as

$$
\begin{aligned}
& \sum_{c \in \Omega} \mathbf{u}_{c} \cdot \frac{d\left(\rho_{c} \mathbf{u}_{c}\right)}{d t} V_{c}+\sum_{c \in \Omega} \mathbf{u}_{c} \cdot \sum_{f \in F(c)} \phi_{f} \hat{M}_{f}=-\sum_{c \in \Omega} \mathbf{u}_{c} \cdot \sum_{f \in F(c)} p_{f} \hat{\mathbf{n}}_{f} A_{f} \\
& +\sum_{c \in \Omega} \mathbf{u}_{c} \cdot \sum_{f \in F(c)} \mu_{f}\left[\left(\mathbf{u}_{n b}-\mathbf{u}_{c}\right) \frac{A_{f}}{\delta d_{f}}+\nabla^{T} \mathbf{u}_{f} \cdot \hat{\mathbf{n}}_{f} A_{f}\right]+\sum_{c \in \Omega} \mathbf{u}_{c} \cdot \mathbf{S}_{c} V_{c}
\end{aligned}
$$

where terms from left to right correspond to time derivative, convection, pressure, diffusion and source contributions to the kinetic energy equation.

Making use of two important identities involving combinations of interpolation and differentiation operators, presented by Felten and Lund [5], and noticing that 
interior fluxes cancel out, Eq. 19 can be rewritten as

$$
\begin{aligned}
& \sum_{c \in \Omega} \frac{d\left(\frac{1}{2} \rho_{c} \mathbf{u}_{c} \cdot \mathbf{u}_{c}\right)}{d t} V_{c}+\sum_{f \in F(\partial \Omega)} \frac{1}{4} \mathbf{u}_{a} \cdot\left(4 \phi_{f} \rho_{f}-\boldsymbol{\phi}_{a} \rho_{a}\right) \hat{U}_{f} A_{f}= \\
& -\sum_{f \in F(\partial \Omega)} \frac{1}{2}\left(\mathbf{u}_{a} p_{f}+\mathbf{u}_{f} p_{a}\right) \cdot \hat{\mathbf{n}}_{f} A_{f}+\sum_{c \in \Omega} p_{c} \sum_{f \in F(c)} \frac{\delta t}{\rho_{f}}\left[\left(p_{n b}-p_{c}\right) \frac{A_{f}}{\delta d_{f}}\right] \\
& -\sum_{c \in \Omega} p_{c} \sum_{f \in F(c)} \frac{\delta t}{2 \rho_{f}}\left[\frac{1}{V_{c}} \sum_{f \in F(c)} p_{f} \hat{\mathbf{n}}_{f} A_{f}+\frac{1}{V_{n b}} \sum_{f \in F(n b)} p_{f} \hat{\mathbf{n}}_{f} A_{f}\right] \cdot \hat{\mathbf{n}}_{f} A_{f} \\
& +\sum_{c \in \Omega} \mathbf{u}_{c} \cdot \sum_{f \in F(c)} \mu_{f}\left[\left(\mathbf{u}_{n b}-\mathbf{u}_{c}\right) \frac{A_{f}}{\delta d_{f}}+\nabla^{T} \mathbf{u}_{f} \cdot \hat{\mathbf{n}}_{f} A_{f}\right]+\sum_{c \in \Omega} \mathbf{u}_{c} \cdot \mathbf{S}_{c} V_{c}
\end{aligned}
$$

which states that in the absence of viscosity $(\mu=0)$ the change in kinetic energy is due to the fluxes through the boundary of the domain, the source terms and a kinetic energy error from the pressure term.

\subsubsection{Staggered kinetic energy conservation}

The staggered kinetic energy equation starts from the staggered momentum equation, Eq. 17. First, the summation over faces is recasted as a summation over cells, second, the resulting equation is multiplied by velocity, $\mathbf{u}$. In this way, the staggered kinetic energy equation is shown to obey the same equation as in the collocated case, Eq. 19.

Proceeding similarly as in the collocated analysis, Eq. 19 is rewritten for the staggered case as

$$
\begin{aligned}
& \sum_{c \in \Omega} \frac{d\left(\frac{1}{2} \rho_{c} \mathbf{u}_{c} \cdot \mathbf{u}_{c}\right)}{d t} V_{c}+\sum_{f \in F(\partial \Omega)} \frac{1}{4} \mathbf{u}_{a} \cdot\left(4 \phi_{f} \rho_{f}-\phi_{a} \rho_{a}\right) \hat{U}_{f} A_{f}= \\
& -\sum_{f \in F(\partial \Omega)} \frac{1}{2}\left(\mathbf{u}_{a} p_{f}+\mathbf{u}_{f} p_{a}\right) \cdot \hat{\mathbf{n}}_{f} A_{f} \\
& +\sum_{c \in \Omega} \mathbf{u}_{c} \cdot \sum_{f \in F(c)} \mu_{f}\left[\left(\mathbf{u}_{n b}-\mathbf{u}_{c}\right) \frac{A_{f}}{\delta d_{f}}+\nabla^{T} \mathbf{u}_{f} \cdot \hat{\mathbf{n}}_{f} A_{f}\right]+\sum_{c \in \Omega} \mathbf{u}_{c} \cdot \mathbf{S}_{c} V_{c},
\end{aligned}
$$

which states that in the absence of viscosity $(\mu=0)$ the change in kinetic energy is due to the fluxes through the boundary of the domain and the source terms.

\section{Numerical tests}

\subsection{Three-dimensional vortex}

The conservation properties are numerically verified by solving a threedimensional vortex. This problem is chosen since is inherently unsteady but at 
the same time has zero net mass flux at the boundaries. The spatially periodic set of three-dimensional vortices shown in Fig. 1 are described by

$$
\begin{aligned}
& u=-A \sin (k x) \cos (k y) e^{-2 k^{2} \nu t}, \\
& v=A \cos (k x) \sin (k y) e^{-2 k^{2} \nu t}, \\
& w=-A,
\end{aligned}
$$

where $A=1.0 \times 10^{3} \mathrm{~m} / \mathrm{s}$ is the velocity amplitude, $k=1$ is the wave number and $\nu=0$ is the kinematic viscosity set to zero.

The vortex is solved in a box of side $2 \pi \times 2 \pi \times 2 \pi$ meshed with 66000 triangular prisms, generated by the constant-step extrusion of a two-dimensional grid (2200 triangles $\times 30$ planes, corresponding to a mesh size of $h=0.2$ ). Moreover, the box is filled with two different fluids; i.e. fluid with density $\rho_{1}=1 \mathrm{~kg} / \mathrm{m}^{3}$ occupies the entire cube except for a sphere of radius $R=\pi / 2$, fixed in the center, that corresponds to a fluid with different densities $\rho_{2}=10,100,1000 \mathrm{~kg} / \mathrm{m}^{3}$. A constant time step of $\Delta t=1.0 \times 10^{3} \mathrm{~s}$ is used. Boundaries $X$ and $Y$ are considered slip walls while periodic conditions are set for $Z$ ones.

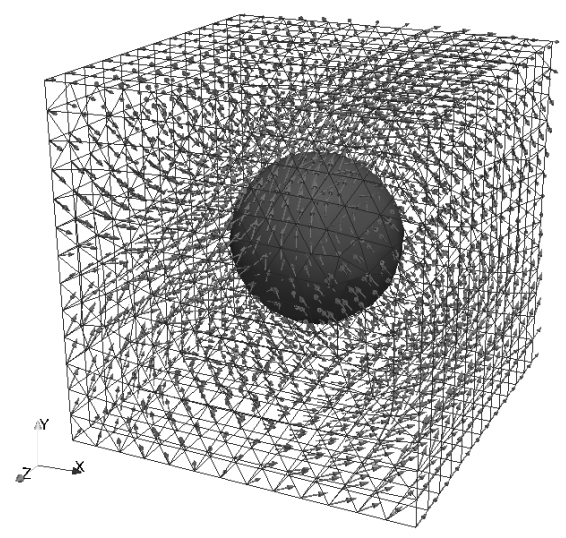

Figure 1: Schematic drawing of the three-dimensional vortex test.

Mass and total momentum for each mesh scheme and sphere density, $\rho_{2}$, are calculated at every time step using Eq. 15 and 16. Results corroborate that both collocated and staggered schemes conserve mass and total momentum as theoretically expected.

This particular test is really appropriate to study the conservation of kinetic energy since viscosity is set to zero, there is no net mass flux at the boundaries and no source terms exist. Under these conditions, the continuous transport equation for kinetic energy, Eq. 18, determines that the rate of change of total kinetic energy is zero, $\partial k / \partial t=\partial\left(\frac{1}{2} \rho \mathbf{u} \cdot \mathbf{u}\right) / \partial t=0$. Hence, if any variation of kinetic energy exists is due to an improper mesh discretization. In this way, the rate of change of 
kinetic energy, $d k / d t$, convection, $\nabla \cdot\left[\mathbf{u}\left(\frac{1}{2} \rho \mathbf{u} \cdot \mathbf{u}\right)\right]$, and pressure, $\nabla \cdot(p \mathbf{u})$, terms for each mesh scheme and sphere density, $\rho_{2}$, are calculated at every time step using Eq. 19. Results confirm that the use of the symmetry-preserving convective scheme turns out in a zero contribution to the energy equation. Otherwise, the pressure term behaves differently depending on the mesh discretization. In detail, staggered pressure contribution is zero for all density ratios, however, the collocated one presents a non-zero value. As an example, kinetic energy rate of change and pressure term are plotted in Fig. 2 for density ratio $\Delta \rho=\rho_{2} / \rho_{1}=10$. The figure clearly shows that the staggered kinetic energy variation is zero, while the collocated case presents and unphysical kinetic energy variation equal to the pressure error term.

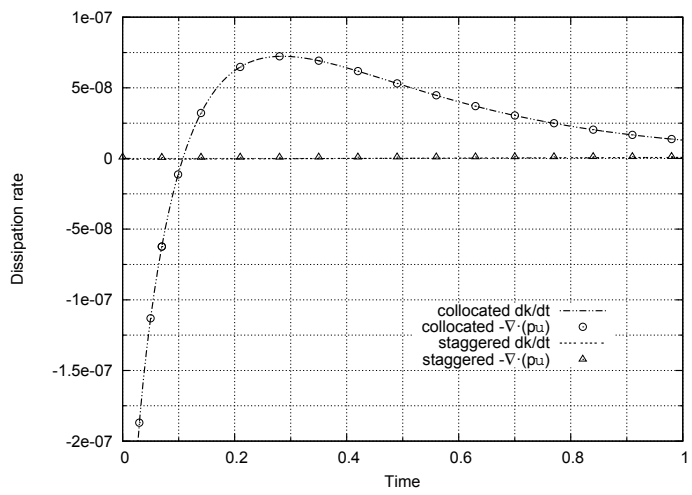

Figure 2: Kinetic energy rate of change and pressure term, using the collocated and staggered mesh schemes, versus time with $\Delta \rho=10$.

It is interesting to numerically study the scaling order of this kinetic energy error intrinsic to the collocated mesh scheme which depends on mesh size and time integration, as previously analyzed in Sec. 3.3.1. First, the comparison between the pressure error term and mesh size is evaluated by solving the vortex, for the three different density ratios, on four successively refined meshes ( $h=0.4$ to $h=0.05$ ) with a fixed time step $\Delta t=1.0 \times 10^{-3} \mathrm{~s}$. Second, the relation between the pressure error term and the time integration is analyzed by solving the same test on the $h=0.2$ mesh, while trying four different time steps $\left(1.0 \times 10^{-2}\right.$ to $\left.5 \times 10^{-5}\right)$.

Results of the kinetic energy pressure error at the first time iteration, depending on the mesh size, are plotted in Fig. 3. The figure shows that if the mesh is refined, the kinetic energy pressure error is reduced in a second-order manner independently of the density ratio. Furthermore, the error difference between density ratios is explained by the density-scaled pressure fields generated by the pressure Poisson equation, Eq. 7. 


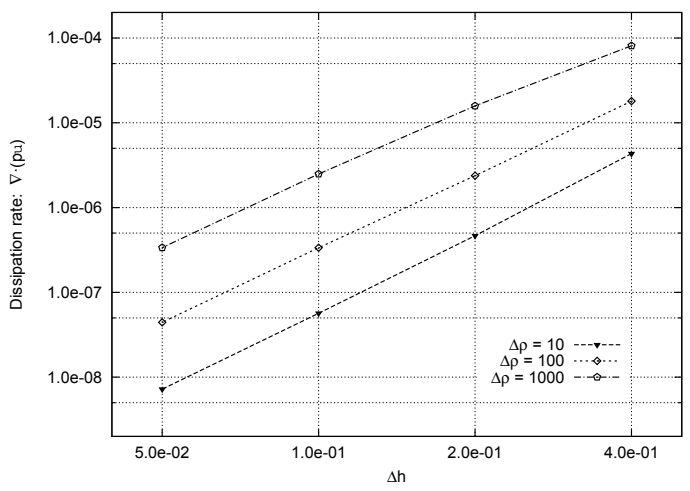

Figure 3: Kinetic energy pressure error for the collocated mesh scheme versus mesh size, for different density ratios.

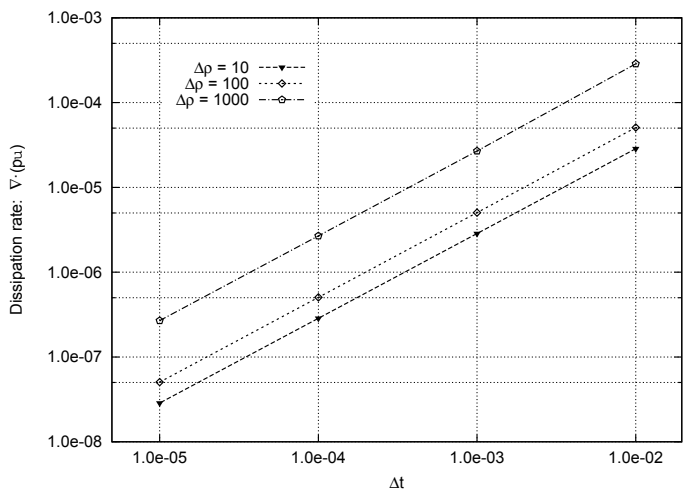

Figure 4: Kinetic energy pressure error for the collocated mesh scheme versus time step, for different density ratios.

The time integration study is plotted in Fig. 4. Results of the kinetic energy pressure error, at the first time iteration, conclude that successively smaller time steps provide proportionally reduced errors. Once again, the error difference between density ratios is due to the different pressure fields obtained. Furthermore, a first-order explicit time integration method has been used for the analysis of the kinetic energy conservation, Sec. 3.3.1, but, using other time integration methods may decrease the kinetic energy error. For example, if using gear like time integration schemes, the time step multiplying the pressure error term in Eq. 20 is diminished by a scaling factor ( $2 / 3$ for a second-order case), therefore, the pressure error term is consequently minimized [6]. 


\subsection{Exact sinusoidal function}

Accuracy of the two mesh schemes is studied by solving an exact sinusoidal function. In each case, a sinusoidal function is assigned to the input variables; i.e. cell-centered velocities, $\mathbf{u}$, in the collocated case while normal face velocities, $U$, in the staggered one. Then, numerical normal face velocities for the collocated case are obtained from Eq. 10, considering the ideal situation where pressure terms vanish and dividing by face density, while numerical cell-centered velocities are calculated from Eq. 14 for the staggered case, respectively. Finally, the root square mean error (rms), $x_{r m s}$, is calculated by comparing analytical and numerical results.

The stream function is set to be $\psi=\frac{1}{2 \pi N} \sin (2 \pi N x) \cos (2 \pi N y) \mathbf{k}$. Thus, instead of changing the mesh size, mesh refinement is performed by changing the wavelength of the input sine functions. The test is performed in a cube of side $1.0 \times 1.0 \times 1.0$ meshed with 9676 tetrahedra. Similarly to the previous test, fluid with density $\rho_{1}=1 \mathrm{~kg} / \mathrm{m}^{3}$ occupies the entire cube except for a sphere of radius $R=0.15$, fixed in the center, filled with a fluid that presents different densities $\rho_{2}=10,100,1000 \mathrm{~kg} / \mathrm{m}^{3}$.

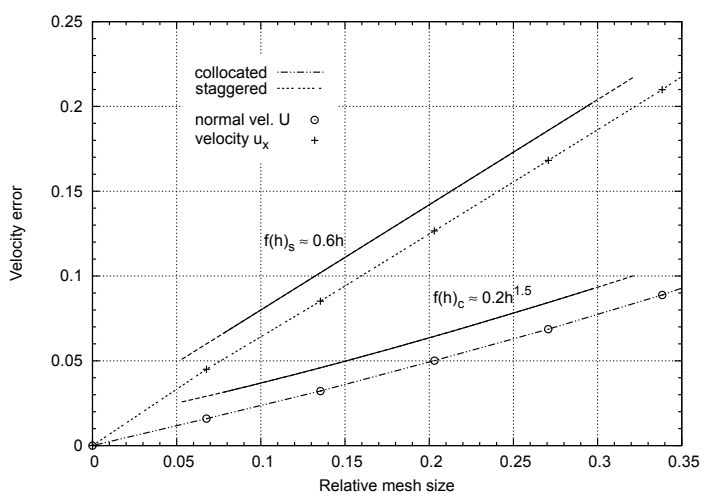

Figure 5: Velocity error versus relative mesh size with $\Delta \rho=10$. Approximated regression equations are calculated.

Errors of $U$ and $u_{x}$ velocity accuracy are obtained for relative mesh sizes ranging from 0 to 0.35 and plotted in Fig. 5 for density ratio $\Delta \rho=\rho_{2} / \rho_{1}=10$; i.e. results come out to be independent of the density ratio. Collocated normal face velocity errors are smaller than staggered cell-centered velocity ones for all relative mesh sizes. In detail, it is observed that collocated errors are almost second-order, $f(h)_{c}=0.2 h^{1.5}$, while staggered ones are just first-order, $f(h)_{s}=0.6 h$. 


\section{Conclusions}

This work presents a collocated and staggered mesh discretizations of the immiscible multiphase Navier-Stokes equations and analyzes their conservation properties and accuracy.

First, a three-dimensional vortex is solved to numerically study the conservation properties. On the one hand, the staggered scheme preserves mass, momentum and kinetic energy. On the other hand, the collocated scheme conserves mass and momentum, but presents a kinetic energy error of the form $\mathcal{O}\left(\Delta t^{m}, \Delta h^{2}, \Delta \rho\right)$, due to the improper pressure gradient formulation.

Second, an accuracy study has been performed by comparing numerical results to the analytical solution of an exact sinusoidal function. Results show that collocated normal face velocity errors (2nd-order) are smaller than staggered cellcentered velocity ones (1st-order) for all relative mesh sizes, considering the ideal situation where pressure terms in Eq. 10 vanish.

\section{Acknowledgements}

This work has been financially supported by the Ministerio de Economía y Competitividad, Spain (ENE-2011-28699), a FPU grant by the Ministerio de Educación, Cultura y Deporte, Spain (AP-2008-03843) and by the collaboration project between the Technical University of Catalonia and Termo Fluids S.L. Calculations have been performed at the Barcelona Supercomputing Center (BSC).

\section{References}

[1] S. P. MacLachlan, J. M. Tang, and C. Vuik. Fast and Robust Solvers for Pressure-Correction in Bubbly Flow Problems. Journal of Computational Physics, 227:9742-9761, 2008.

[2] M. Raessi and H. Pitsch. Consistent Mass and Momentum Transport for Simulating Incompressible Interfacial Flows with Large Density Ratios Using the Level Set Method. Computers and Fluids, 63:70-81, 2012.

[3] B. Perot. Conservation Properties of Unstructured Staggered Mesh Schemes. Journal of Computational Physics, 159:58-89, 2000.

[4] R. W. C. P. Verstappen and A. E. P. Veldman. Symmetry-preserving discretization of turbulent flow. Journal of Computational Physics, 187(1):343-368, 2003.

[5] F. N. Felten and T. S. Lund. Kinetic Energy Conservation Issues Associated with the Collocated Mesh Scheme for Incompressible Flow. Journal of Computational Physics, 215(2):465-484, 2006.

[6] L. Jofre, O. Lehmkuhl, J. Ventosa, and A. Oliva. Conservation Properties and Accuracy of Unstructured Mesh Schemes for the Navier-Stokes Equations. In Proceedings of the Turbulence, Heat and Mass Transfer 7, pages 1-12, 2012. 